\title{
Effects of instantaneous object input and past experience on object-based attention
}

\author{
Ming-Chou Ho ${ }^{a}$, Su-Ling Yeh ${ }^{\mathrm{b}, *}$ \\ ${ }^{a}$ Department of Psychology, Chung-Shan Medical University, Taichung, Taiwan \\ ${ }^{\mathrm{b}}$ Department of Psychology, National Taiwan University, No. 1, Sec. 4, Roosevelt Rd., Taipei 106, Taiwan
}

\section{A R T I C L E I N F O}

\section{Article history:}

Received 12 September 2008

Received in revised form 4 February 2009

Accepted 5 February 2009

Available online 17 March 2009

\section{PsyclNFO classification: \\ 2323}

2346

Keywords:

Location

Validity

Probability

Top-down

Bottom-up

\begin{abstract}
A B S T R A C T
Evidence for object-based attention typically comes from studies using displays with unchanged objects, and no consensus has yet been reached as to whether the object effect would be altered by changing object displays or having seen this change across-trials. We examined this by using modifications of the double-rectangle cuing paradigm of Egly et al. [Egly, R., Driver, J., \& Rafal, R. D. (1994). Shifting visual attention between objects and locations: Evidence from normal and parietal lesion subjects. Journal of Experimental Psychology: General, 123, 161-177], and our results, when the objects remained unchanging, replicated the original object effect. However, no object effect was found when the rectangles disappeared from view in the last (target) frame. This was true regardless of the likelihood of the rectangles disappearing, indicating the importance of instantaneous object inputs for object-based attention. The across-trial experience of seeing a different object (boomerang), however, was found to influence the object effect when the cued rectangles persisted throughout the trial. Unlike previous studies, which emphasize one or the other, we demonstrate clearly that instantaneous object inputs and past experience interact to determine the way attention selects objects.
\end{abstract}

(c) 2009 Elsevier B.V. All rights reserved.
Due to the limited capacity of our cognitive system, selection is necessary to prevent overloading. Past studies have shown that the units of selection can be spatial locations (e.g., Eriksen \& Yeh, 1985; Posner, Snyder, \& Davidson, 1980), as well as discrete objects (e.g., Duncan, 1984; Vecera, 1994). In a seminal paper, Egly, Driver, and Rafal (1994) used a cueing paradigm with a now popular doublerectangle display to demonstrate the existence of object-based attention (OBA). In this paradigm, typical displays include two outlined rectangles that are shown throughout, and one end of one of the rectangles is brightened as a cue to indicate the possible location of a target. The target is a small solid square, shown subsequently within one end of a rectangle. OBA is indicated by the object effect: that is, RTs are shorter for an invalidly cued target (one which appears at the uncued end of the rectangle) than for the uncued rectangle, with an equal cue-to-target distance between the two.

Many researchers have adopted this paradigm to investigate various properties of OBA (for a review, see Scholl, 2001). Given that most studies using this paradigm use unchanging objects, little is known about whether OBA is affected by the disappearance or change of the object after it has been cued, although some inferences can be drawn. On the one hand, when attention is cued to the target object and selects it for further processing, it should be this

\footnotetext{
* Corresponding author. Tel.: +886 2 33663097; fax: +886 223629909 .

E-mail address: suling@ntu.edu.tw (S.-L. Yeh).
}

cued object that forms the object representation (Goldsmith \& Yeari, 2003), which remains selected as a processing unit. Thus, it is reasonable to hypothesize that it is the originally cued object ${ }^{1}$ that determines OBA (i.e., the cued object hypothesis). On the other hand, however, the retinal inputs of our visual world change constantly, and so do our experiences and behavioral goals. Our visual system should thus reflect these complexities; namely, where the object changes or disappears after the cue display, it should be the change, rather than the originally cued object, that determines OBA (i.e., the dynamic updating hypothesis).

Our first goal in this study was to test these two hypotheses, by making the cued objects disappear (Experiment 1 ) or by replacing them with a very different object (Experiments 2 and 3 ) in the target frame. The critical manipulations took place in the target frame: before that point, the two parallel rectangles remained unchanged, as in the typical Egly et al.'s display. We predicted that if the cued object hypothesis is true, a typical object effect would be observed even when the originally cued rectangles disappeared in

\footnotetext{
1 Our main focus was to examine whether it is the originally cued object that determines OBA, rather than which representations are cued and selected. Past studies have shown that when an object is cued, attention can operate on different representations (e.g., grouped locations formed by the cued object or features pertaining to the cued object) depending on task demands (e.g., Lamy \& Tsal, 2000; Vecera \& Farah, 1994). In the current study, no matter what representations are selected, once the selected representations determine OBA, the cued object hypothesis is supported.
} 
the target frame (Experiment 1). On the other hand, if the dynamic updating hypothesis is true, no object effect would be observed in this case. Furthermore, if the cued object hypothesis is true, then when the originally cued rectangles were replaced suddenly by a boomerang shape in the target frame (Experiments 2 and 3), a typical object effect based on the cued rectangles should be observed, regardless of the presence of the boomerang. In contrast, according to the dynamic updating hypothesis, the object effect should be affected by the presence of the boomerang.

Our second goal was to examine how top-down influences interplay with the object inputs to determine OBA, by manipulating the probability of disappearance or replacement of the objects. Many studies have shown that expectation (Jefferies, Wright, \& Di Lollo, 2005; Shomstein \& Yantis, 2002; Shomstein \& Yantis, 2004) modulates attentional control in a scene which determines attentional allocation even for attentional capture by task-irrelevant distractors (Atchley, Kramer, \& Hillstrom, 2000; Folk \& Remington, 1999; but see Yeh \& Liao, 2008). Although it is undeniable that bottom-up stimulus inputs are important as well, past studies do seem to suggest that top-down influence ultimately determines human behaviors (e.g., Bacon \& Egeth, 1994; Chen, 1998; Downing, 2000; Farah, 1985; Farah, 1989; Folk, Remington, \& Johnston, 1992; Lamy \& Tsal, 1999; Li \& Logan, 2008). We test whether this is also true for OBA. If expectation (created by manipulating the probability of object disappearance in Experiment 1 or object replacement in Experiments 2 and 3 ) affects OBA, then we should expect to find the observed object effect correlated with the probability of object presence.

Creating expectation by means of probability manipulation could bias participants' selection toward the objects if the objectpresence probability is high rather than low. That is, when there is a high probability that an object will appear, participants are more likely to "utilize" this object to guide their detection of the target (e.g., they may follow the contour of the objects to search for the target). Thus, for the high probability trials in which rectangles would appear more frequently throughout a trial (e.g., an $80 \%$ chance of appearing), participants would be more predisposed to use these rectangles to guide their detection. On the other hand, when the rectangles are less likely to appear (e.g., a 20\% chance), participants would be less likely to use them for detection. Therefore, we hypothesize a larger object effect in the higher probability condition. However, while considering the role of expectation in OBA, we should not neglect the bottom-up factors (e.g., sudden change of the scene). That is, the top-down effect of expectation could be overridden by the bottom-up inputs.

\section{Experiment 1}

The rectangles were presented until the onset of the target for $20 \%$ of total trials in Experiment $1 \mathrm{~A}$ and for $80 \%$ of the total trials in Experiment 1B. Otherwise, they remained present throughout the trial. In the rectangle-absent trials, the previously presented rectangles disappeared and left only the target (a square) in the target-present trials, and an empty space in the target-absent (catch) trials. According to the cued object hypothesis, once the object is cued and sufficient time is given for the object to be selected, the object effect should be observed. Additionally, if expectation does affect OBA, the object effect should interact with the probability of object appearance.

\subsection{Method}

\subsubsection{Participants}

All participants in this study were undergraduates of the National Taiwan University, with normal or corrected-to-normal vi- sion. There were 25 participants in Experiment $1 \mathrm{~A}$ and 25 in Experiment 1B.

\subsubsection{Apparatus, design, and procedure}

The stimuli were constructed with and controlled by the Eprime software (Schneider, Eschman, \& Zuccolotto, 2002), and were presented on a 17-inch calibrated View Sonic color monitor (at a refresh rate of $85 \mathrm{~Hz}$ ) in a dimly lighted chamber. The participant placed his/her head on a chin rest and viewed the stimuli binocularly at a distance of $80 \mathrm{~cm}$.

Each trial began with a central fixation (a plus sign) and two vertical rectangles $\left(2^{\circ} \times 16^{\circ}\right.$, color grey) at the $7^{\circ}$ eccentric locations on the left and right side of the fixation point, presented for $1000 \mathrm{~ms}$ (Fig. 1). Using only vertical rectangles, rather than vertical and horizontal, allowed us to keep the experiment at a reasonable length of time (about $40-50 \mathrm{~min}$ ) so that participants could finish it in a single visit. Also, studies using both vertical and horizontal rectangles have not found any orientation-related changes in the size of the object effect (e.g., Brown \& Denney, 2007; Egly et al., 1994).

Because the spatial extent of the initial focusing of attention modulates the object effect (Goldsmith \& Yeari, 2003), we added a pre-cue event for $35 \mathrm{~ms}$ (four grey L-shapes, $3^{\circ} \times 3^{\circ}$, surrounding the two rectangles) which served to expand the focus of attention to cover both rectangles. The pre-cue event was added in order to insure that the participants' attention would cover all the objects in the display; otherwise the absence of the object effect could have been attributed to the lack of wide-enough attention for object representations to be formed. Although a peripheral cue, as we have used here, is assumed to induce spread attention and lead to the object effect (Goldsmith \& Yeari, 2003), it is always possible that the absence of the object effect, should it happen, could be attributed to the pre-focusing of attention (not explicitly manipulated) rather than to the factor that is manipulated. As our pilot studies indicated that no object effect was observed in the object-absent condition, this pre-cue event was necessary to exclude the possibility that a lack of attention spread could lead to the absence of the object effect.

The distance between the corner of each $\mathrm{L}$ and the central fixation was $14.6^{\circ}$. Seventy milliseconds after the offset of the four Ls, a $\Pi$-shaped red cue (outlining one end of the rectangle) was presented for $100 \mathrm{~ms}$, followed by a $200 \mathrm{~ms}$ fixation display, and then the target (a grey square, $2^{\circ} \times 2^{\circ}$ ). In the target frame, the two rectangles could either disappear (Fig. 1A) or remain along with the target. The target remained on screen until either the participant responded or 2 seconds elapsed, and then the next trial began. Any response to the catch trials that contained no target was recorded as an error, as was the failure to respond. Sound feedback was given when the participants responded to the catch trials.

The target appeared at the cued location in $76 \%$ of trials (this was the valid condition). In the remaining $24 \%$ invalid trials, the target could appear at either the uncued location occupied by the cued object (12\%; this was the invalid-same condition) or at an equidistant location on an uncued object (12\%; the invalid-different condition). In Experiment $1 \mathrm{~A}$, the rectangles appeared in the target frame in $20 \%$ of trials ( 120 trials; 76 valid, 24 invalid, and 20 catch trials) and were absent in the remaining 80\% (480 trials; 304 valid, 96 invalid, and 80 catch trials). In Experiment 1B, the rectangles appeared in the target frame for $80 \%$ of trials ( 480 trials; 304 valid, 96 invalid, and 80 catch trials) and were absent in the remaining $20 \%$ ( 120 trials; 76 valid, 24 invalid, and 20 catch trials). The same probability was applied for the practice trials ( 6 object-present trials and 24 object-absent trials in Experiment 1A, reversed in Experiment 1B). All conditions (valid, invalid-same, invalid-different, and catch trials) were randomized within a single block, and breaks were given every 40 trials. 
(A) Rectangles are removed

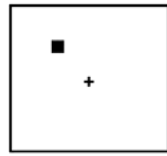

Valid

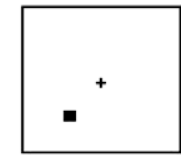

Invalid-same

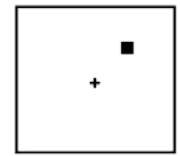

Invalid-different

(B) Rectangles are replaced with a boomerang

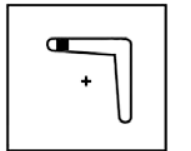

Valid

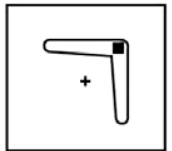

Invalid-same

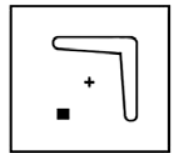

Invalid-different

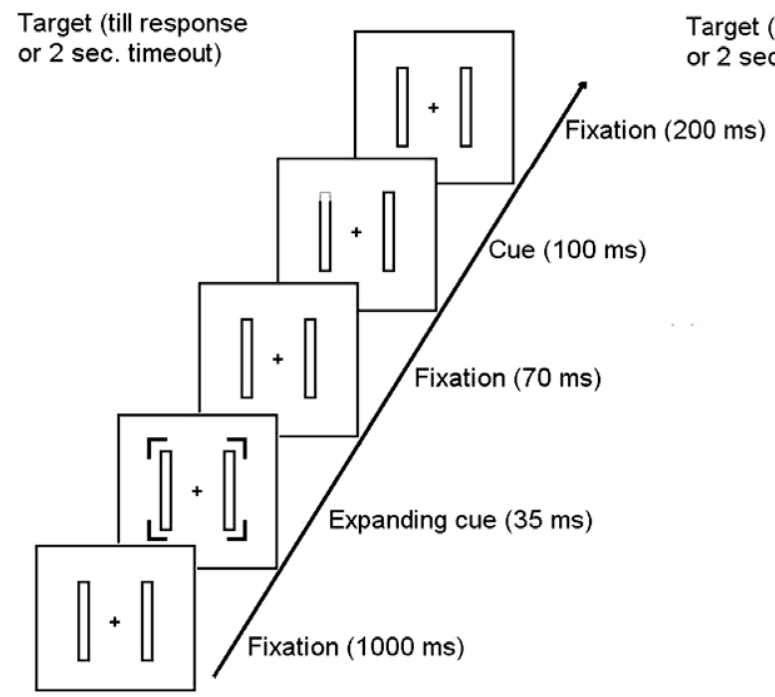

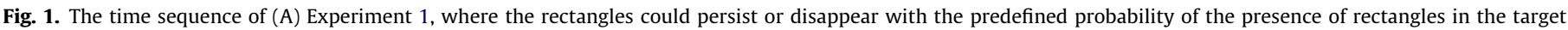

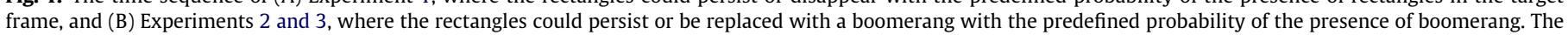
four expanding signals and the target were grey in the experiment, and the cue was red.

\section{Results and discussion}

The false alarm rate was $2.6 \%$ and the miss rate was $.05 \%$ in Experiment $1 \mathrm{~A}$, and, respectively, they were $3.2 \%$ and $.2 \%$ in Experiment 1B. Correct RTs faster than $150 \mathrm{~ms}$ and beyond three standard deviations from the mean RT in each condition were removed, which resulted in $2.0 \%$ and $1.7 \%$ removal rates in Experiments $1 \mathrm{~A}$ and $1 \mathrm{~B}$, respectively.

Correct RTs (Table 1) in Experiments $1 \mathrm{~A}$ and $1 \mathrm{~B}$ were submitted to a three-way ANOVA of object-presence probability (20\% or $80 \%) \times$ object (presence or absence) $\times$ target location (valid, invalid-same, or invalid-different), with the first as a between-subject factor and the other two as within-subject factors. The main effects of object $(F(1,48)=121.94$, MSe $=339.9, p<.0001)$ and target location $(F(2,96)=250.78$, MSe $=290.7, p<.0001)$ were significant. That is, RTs were faster when the objects were present than when they were absent, and post hoc comparison indicates that target detection was faster when the target was at the valid location than when at the invalid-same and invalid-different locations.

The three-way interaction was not significant, but the following two-way interactions were: probability $\times$ object $(F(1,48)=49.92$, MSe $=339.9, \quad p<.0001), \quad$ probability $\times$ target location $(F(2,96)=5.51, \mathrm{MSe}=290.7, p<.01)$, and object $\times$ target location $(F(2,96)=21.50, \mathrm{MSe}=144.9, p<.0001)$. Further analyses of the first two interaction effects showed that RTs in the object-present condition were shorter than those in the object-absent condition, regardless of the probability of the presence of the rectangles. Moreover, RT difference between the object-present and object-absent conditions was larger in the $80 \%$ probability condition $(39 \mathrm{~ms})$ than that in the $20 \%$ probability condition $(8 \mathrm{~ms})$. With $80 \%$ probability, all pairs, with the exception of the difference between invalid-same and invalid-different locations, were significant. With 20\% probability, all paired comparisons were significant.
More importantly, for our main purpose here (being to test the two hypotheses by examining whether the object effect would be affected by manipulating probability,) we further analyzed the interaction of object $\times$ target location. The simple main effects indicated that when objects were present, all paired comparisons were significant (all $p$ 's $<.0001$ ). The significant RT difference (10 ms) between the invalid-same and invalid-different locations indicated the presence of the object effect. When objects were absent, the difference between invalid-same and invalid-different conditions was not significant, indicating that the object effect was also absent (RT difference $=2 \mathrm{~ms}, p=.477, \eta_{p}^{2}=.01$ ). These results indicate that regardless of probability manipulation, the occurrence of the object effect was associated with the presence of objects in the target frame, supporting the dynamic updating hypothesis. These results argue against the cued object hypothesis, because even when the objects were cued previously, the object effect was negligible if they were absent in the target frame. In addition, expectation did not seem to influence OBA because the object effect did not interact with the manipulated probability, although it did affect the overall RT advantage of the unchanged vs. changed displays.

One might suspect that the pre-cue event (i.e., the four grey Ls presented before the cue) may have in fact interfered with participants' attention to the original rectangle objects, because it may have introduced several new "objects" through their abrupt onset, just prior to the appearance of the peripheral cue. Considering that a significant object-based effect was still found in Experiments $1 \mathrm{~A}$ and $1 \mathrm{~B}$; however, the Ls apparently did not prevent attention to the rectangles, and thus should not affect our main conclusion. Moreover, since no object effect was observed in the object-absent condition, regardless of probability, the pre-cue event was proven to be necessary in order to ensure wide-enough attention to cover the objects (Goldsmith \& Yeari, 2003). 
Table 1

Mean RTs (in ms) and standard errors (in parentheses) in this study.

\begin{tabular}{|c|c|c|c|c|c|c|}
\hline \multirow[t]{2}{*}{ Experiment } & \multirow[t]{2}{*}{ Rectangle-presence probability (\%) } & \multirow[t]{2}{*}{ Object in target frame } & \multicolumn{3}{|l|}{ Validity } & \multirow[t]{2}{*}{ Object effect } \\
\hline & & & Valid & Invalid-same & Invalid-different & \\
\hline $1 \mathrm{~A}$ & 20 & $\begin{array}{l}\text { None } \\
\text { Rectangles }\end{array}$ & $\begin{array}{l}371(9) \\
373(10)\end{array}$ & $\begin{array}{l}417(10) \\
400(11)\end{array}$ & $\begin{array}{l}420(10) \\
410(12)\end{array}$ & n.s. \\
\hline 1B & 80 & $\begin{array}{l}\text { None } \\
\text { Rectangles }\end{array}$ & $\begin{array}{l}376(7) \\
350(6)\end{array}$ & $\begin{array}{l}439(7) \\
389(7)\end{array}$ & $\begin{array}{l}439(8) \\
399(7)\end{array}$ & n.s. \\
\hline $2 \mathrm{~A}$ & 20 & $\begin{array}{l}\text { Rectangles } \\
\text { Boomerang }\end{array}$ & $\begin{array}{l}396(8) \\
400(8)\end{array}$ & $\begin{array}{l}425(10) \\
416(8)\end{array}$ & $\begin{array}{l}432(10) \\
453(9)\end{array}$ & n.s. \\
\hline $2 \mathrm{~B}$ & 80 & $\begin{array}{l}\text { Rectangles } \\
\text { Boomerang }\end{array}$ & $\begin{array}{l}356(8) \\
436(10)\end{array}$ & $\begin{array}{l}387(9) \\
439(14)\end{array}$ & $\begin{array}{l}393(11) \\
492(9)\end{array}$ & n.s. \\
\hline $3 \mathrm{~A}$ & 20 & $\begin{array}{l}\text { Rectangles } \\
\text { Boomerang }\end{array}$ & $\begin{array}{l}392(9) \\
385(11)\end{array}$ & $\begin{array}{l}409(10) \\
420(12)\end{array}$ & $\begin{array}{l}421(10) \\
453(13)\end{array}$ & * \\
\hline $3 B$ & 80 & $\begin{array}{l}\text { Rectangles } \\
\text { Boomerang }\end{array}$ & $\begin{array}{l}352(9) \\
410(13)\end{array}$ & $\begin{array}{l}376(10) \\
447(13)\end{array}$ & $\begin{array}{l}388(10) \\
484(15)\end{array}$ & * \\
\hline
\end{tabular}

n.s.: Not significant statistically, $p>.05$.

: Significant statistically, $p<.05$.

In summary, in this experiment, the rectangles disappeared in the target frame in some proportion of trials. We found that the probability of the presence of rectangles could affect the overall patterns of target detection RT, as indicated by the interactions of probability $\times$ object and probability $\times$ target location. However, it had no impact on the occurrence of the object effect. The attentional resources directed by the exogenous cue to the cued and presumably attended rectangle may have been quickly "released" when the rectangles disappeared. In other words, when an object disappears, there is simply no object in the representation upon which attention can focus (Miller, 1989), even with a low probability and thus a low expectation of such a sudden disappearance.

\section{Experiment 2}

The previous experiment showed clearly that the object effect were associated with the presence of the object in the target frame but not with the cue, supporting the dynamic updating hypothesis over the cued object hypothesis. However, the probability of the object's presence had little effect when object inputs were suddenly truncated. Once they are gone, the object effect are gone as well, regardless of how frequently the objects are seen. Is it possible that "disappearance" is too strong to be affected by expectation? In this experiment, we replaced the rectangles with a different object in the target frame. A boomerang shape was used to replace the original rectangles in a certain proportion of trials ( $80 \%$ or $20 \%$ ). In some trials, the rectangles were present throughout the trial. In others, a boomerang appeared in the target frame, interrupting the appearance of the two rectangles. Unlike in Experiment 1 , there were always objects (either rectangles or a boomerang) present in the target frame.

\subsection{Method}

\subsubsection{Participants}

Sixty-four participants took part in Experiments 2A and 2B, with 32 in each.

\subsubsection{Design and procedure}

In Experiment 2A, the rectangles appeared in the target frame in $20 \%$ of trials ( 120 trials, including 76 valid, 24 invalid, and 20 catch trials) and the boomerang appeared in the remaining $80 \%$ of trials (480 trials; 304 valid, 96 invalid, and 80 catch trials). The definitions of the invalid-same and invalid-different target locations depended on the object shown in the target frame. If two rectangles were shown in the target frame, the definitions were the same as those in Experiment 1. If there was one boomerang in the target frame, the invalid-same target location occurred when the target appeared at the corner of the boomerang, and the invalid-different target location occurred when the target appeared at an equidistant location outside the boomerang (Fig. 2). The size of the boomerang was similar to that of the large square formed by the two rectangles. The same probability of boomerang-presence (80\%) was applied in the practice trials (6 rectangles and 24 boomerang trials).

In Experiment 2B, two rectangles appeared in the target frame in $80 \%$ of trials ( 480 trials; 304 valid, 96 invalid, and 80 catch trials) and one boomerang appeared in the remaining $20 \%$ of trials ( 120 trials; 76 valid, 24 invalid, and 20 catch trials). Again, the same boomerang-presence probability (20\%) was applied in the practice trials (24 rectangles and 6 boomerang trials).

In the boomerang-present trials, the orientation of the boomerang changed with the cue location that was inconsistent with the supposed object effect obtained with the two rectangles. For example, when the cue flashed on the upper end of the left rectangle, the 7 -shaped boomerang would appear in the target frame. The object effect of the boomerang was indexed by comparing the target detection time when the target was at the invalidly cued location on the boomerang (i.e., right corner), as opposed to when it was at an equidistant uncued location outside the boomerang.

The sequence and timing of stimuli in Experiment 2 were the same as those of Experiment 1.

\subsection{Results and discussion}

The false alarm rate was $3 \%$ and the miss rate was $0 \%$ in both Experiments 2A and 2B. The same exclusion criteria as in Experiment 1 resulted in $1.8 \%$ and $2.5 \%$ removal rates in Experiments $2 \mathrm{~A}$ and $2 \mathrm{~B}$, respectively.

We conducted a three-way ANOVA for correct RTs of boomerang-presence probability ( $80 \%$ or $20 \%) \times$ object type (boomerang or rectangles) $\times$ target location (valid, invalid-same, or invalid-different), with the first as a between-subject factor, and the other two as within-subject factors. The main effects of object type $(F(1,62)=281.33, \quad \mathrm{MSe}=577.9, \quad p<.0001)$ and target location $(F(2,124)=119.74, \mathrm{MSe}=553.2, p<.0001)$ were significant. RTs were faster when it was the rectangles rather than the boomerang that appeared in the target frame. In addition, target detection was faster when the target was at the cued location than when at the invalid-same location, which in turn was faster than when at the 


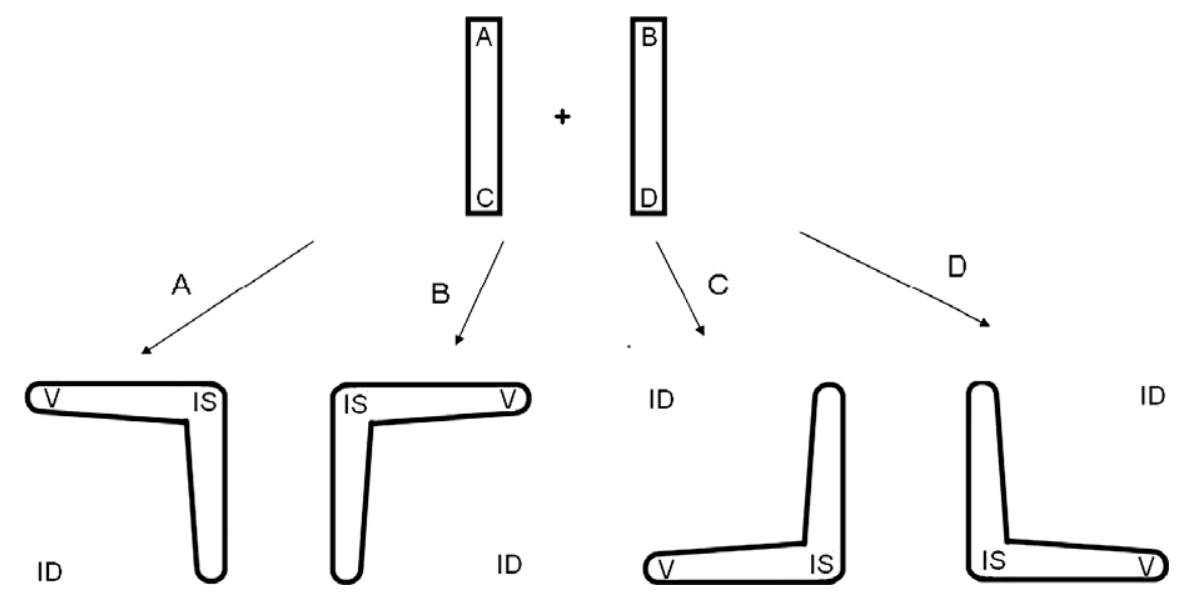

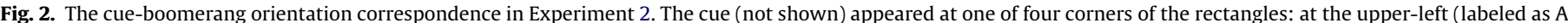

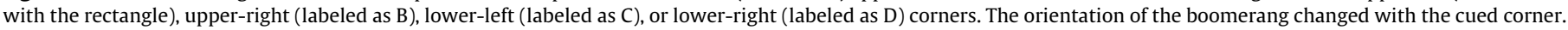

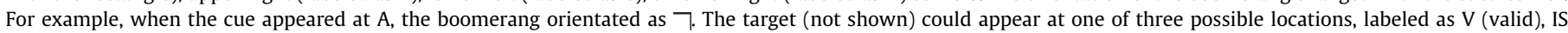
(invalid-same), and ID (invalid-different).

invalid-different location. The three-way interaction was not significant $(p=.471)$. The two-way interactions of object type $\times$ target location $(F(2,96)=28.29$, MSe $=490.00, \quad p<.0001)$ and object type $\times$ boomerang-presence probability $(F(1,48)=172.37$, MSe $=574.0, p<.0001)$ were significant, and thus were analyzed further.

For the interaction of object type $\times$ target location, when the rectangles were present in the target frame, target detection was fastest in the valid location $(376 \mathrm{~ms})$, all $p$ 's $<.0001$. There was no RT difference between the invalid-same ( $406 \mathrm{~ms}$ ) and invaliddifferent (413 ms) target locations $\left(p=.110, \eta_{p}^{2}=.08\right)$, indicating the absence of the object effect. This result also argues against the cued object hypothesis, because even though the rectangles were not only cued but also persisted throughout the trial, no object effect was observed. It is possible that the participants could have learned the correspondence in orientation of the cue and boomerang in previous trials, which could have influenced the online object representations. We examine this possibility further in the next experiment.

When the boomerang was shown in the target frame, target detection was fastest in the valid location $(418 \mathrm{~ms})$, slower in the invalid-same location ( $428 \mathrm{~ms}$ ), and slowest in the invalid-different location ( $472 \mathrm{~ms}$ ), all p's $<.008$. The RT difference between the invalid-same and invalid-different target locations indicates an object effect. Thus, the object effect was obtained only when the boomerang was present in the target frame, not the rectangles, regardless of probability manipulation. This result indicates that the occurrence of the object effect was associated with the presence of the boomerang and based on its orientation, supporting the dynamic updating hypothesis. This result also goes against the cued object hypothesis, because when the rectangles were replaced in the target frame, the object effect was not based on them, even though they were cued previously. Finally, because the object effect did not interact with the manipulated probability when it was the boomerang in the target frame, it seems that expectation of the presence of the boomerang does not seem to influence the object effect based on it.

In the interaction of object type $\times$ boomerang-presence probability, when the object in the target frame had a higher probability of appearing, the participants detected a target on that object more readily. Target detection on the boomerang-present trials was faster when the probability of the boomerang's presence was $80 \%$ than when it was $20 \%$. Similarly, target detection on the rectangle-present trials was faster when the rectangles were more likely to appear in the target frame (20\% boomerang-presence) than when they were less likely to appear ( $80 \%$ boomerang-presence). This indicates that targets appearing on rectangle-present or boomerang-present trials were detected faster when the corresponding trial was expected than when unexpected, although expectation did not impact the object effect for the boomerang in particular.

We think that the across-trial experience is a gradually learned process of the correspondence between the cue and the orientation of the boomerang, which has to be accumulated over previous trials. This possibility can be tested by the following analysis. For each of the probabilities of rectangle-presence $(20 \%$ or $80 \%)$, we calculated the rectangle-based object effect and their effect sizes $\left(\eta_{p}^{2}\right)$ for the first half (300) and the second half (300) of total trials (600). If the learning of the correspondence between the cue and boomerang orientation through previous trials is a gradual process, we predicted that the rectangle-based object effect would be reduced as the trials proceed.

For the 20\% rectangle-presence probability, the object effect was $-2 \mathrm{~ms}\left(\eta_{p}^{2}<.0001\right)$ for the first half, and it was $-1 \mathrm{~ms}$ $\left(\eta_{p}^{2}=.001\right)$ for the second half. For the $80 \%$ rectangle-presence probability, the object effect was $11 \mathrm{~ms}\left(\eta_{p}^{2}=.083, p=.110\right)$ for the first half, and $0 \mathrm{~ms}\left(\eta_{p}^{2}<.0001\right)$ for the second half. We also calculated the object effect and their effect sizes every 100 trials. Results also showed a decreasing effect size as trials proceeded. For the $20 \%$ rectangle-presence probability, the effect sizes were $.013, .104, .007, .001, .009$ and .012 . For the $80 \%$ rectangle-presence probability, the effect sizes were $.063, .013, .105, .037, .015$, and .006 . The reduced effect size for both $20 \%$ and $80 \%$ rectangle-presence probabilities indicates that the across-trial experience of having seen the boomerang could influence the object effects, and this effect is more likely to be gradual.

Although the decreasing effect sizes supported the role of the across-trial experience of having seen the boomerang, an experiment manipulating the boomerang in the opposite way was critical to provide stronger evidence. Therefore, Experiment 3 was designed to manipulate the boomerang orientation in order to induce the object effect when the rectangles were continuously presented until the target frame.

\section{Experiment 3}

In this experiment, the correspondence between the orientations of the cue and boomerang was designed to restore the object 
effect when the rectangles were continuously presented until the target frame. That is, the boomerang orientation was such that it would cause the object effect in a direction consistent with the rectangles. If the learned correspondence of cue and boomerang orientation in previous trials was important to determine OBA, the object effect should remain when it was the rectangles that were present in the target frame.

\subsection{Method}

\subsubsection{Participants}

Forty participants took part in Experiments 3A and 3B, with 20 participants in each.

\subsubsection{Design and procedure}

The design and procedure of Experiments $3 \mathrm{~A}$ and $3 \mathrm{~B}$ were similar to those of Experiments $2 \mathrm{~A}$ and $2 \mathrm{~B}$ except for the cue-boomerang orientation correspondence. When the boomerang appeared in the target frame, its orientation changed with the cue location. Fig. 3 shows the cue location, the corresponding boomerang orientation, and the target location. For example, when the cue flashed on the upper end of the left rectangle, the boomerang became the shape of an $\mathrm{L}$ in the target frame. The trial arrangements of Experiments $3 \mathrm{~A}$ and $3 \mathrm{~B}$ were identical to those of Experiments $2 \mathrm{~A}$ and $2 \mathrm{~B}$, respectively. That is, in Experiment $3 \mathrm{~A}$, the boomerang-presence probability was $80 \%$, whereas in Experiment 3B, it was $20 \%$.

\subsection{Results and discussion}

The false alarm, missing, and removal rates were $2.6 \%, .07 \%$, and $1.6 \%$, respectively, in Experiment $3 \mathrm{~A}$; and they were $3 \%, 1 \%$, and $2.8 \%$ in Experiment 3B.

The main effects of object type $(F(1,38)=185.30$, MSe $=615.2$, $p<.0001)$ and target location $(F(2,76)=161.47, \quad \mathrm{MSe}=329.7$, $p<.0001)$ were significant. As in Experiment 2, RTs were longer when the boomerang appeared in the target frame than when the rectangles appeared. In addition, target detection was faster when the target was at the cued location than when at the invalid-same location, and also faster than when it was at the invalid-different location.

The three-way interaction was not significant. Two significant two-way interactions were analyzed further. For the interaction of object type $\times$ boomerang-presence probability $(F(1,38)=98.00$, MSe $=615.2, p<.0001$ ), detecting a target on the rectangle-present trials was faster when the rectangles were more likely to appear in the target frame (20\% boomerang-presence) than when they were less likely ( $80 \%$ boomerang-presence). For the interaction of object type $\times$ target location $(F(2,76)=28.32, \quad M S e=274.7, p<.0001)$, when the rectangles continuously presented until the target frame, target detection was fastest in the valid location (372 ms), slower in the invalid-same location (392 ms), and slowest in the invaliddifferent location (404 ms), all p's $<.0001$. The RT difference between the invalid-same and invalid-different locations $(11 \mathrm{~ms})$ indicated the presence of the object effect. When the boomerang was in the target frame, target detection was fastest in the valid condition $(398 \mathrm{~ms})$, slower in the invalid-same condition (433 ms), and slowest in the invalid-different condition (469 ms), all $p$ 's $<.0001$. Regardless of probability manipulation, the object effect was still obtained when the original rectangles were replaced by a boomerang in the target frame.

To provide statistical support for the statement that the acrosstrial experience of seeing the boomerang plays an important role in the object effect, we conducted a three-way ANOVA on the factors of experiment (2A, 2B, 3A, or 3B), object type (rectangles or boomerang), and target location (valid, invalid-same, or invalid-different). A critical three-way interaction was obtained as expected $(F(6,200)=29.18, \mathrm{MSe}=302.6, p<.0001)$. Further post hoc analysis showed two-way interactions of object type $\times$ target location in each experiment. Analysis of these two-way interactions showed that when the rectangles persisted in the display, the object effect was absent in Experiments $2 \mathrm{~A}\left(p>.3, \eta_{p}^{2}=.033\right)$ and $2 \mathrm{~B}(p>.1$, $\left.\eta_{p}^{2}=.089\right)$, whereas it was observed in Experiments $3 \mathrm{~A}(p<.05$, $\left.\eta_{p}^{2}=.264\right)$ and 3B $\left(p<.005, \eta_{p}^{2}=.396\right)$. The critical significant three-way interaction and its subsequent pair-wise analysis indicated that the across-trial experience of seeing the boomerang did indeed affect the object effect of the rectangles.

\section{General discussion}

By adopting the cueing paradigm of Egly et al. (1994) and causing the objects in the last frame to disappear or be replaced with another object, this study yielded three important findings. First, instantaneous object inputs were found to be critical in the occurrence of the object effect, which supports the dynamic updating hypothesis over the cued object hypothesis. In Experiment 1, we obtained the object effect only when the rectangles remained in the target frame, but not when they disappeared from view. Had the cued object per se been critical, the object effect should have been observed in both cases. Experiment 2 showed that when the boomerang appeared suddenly in the target frame to replace

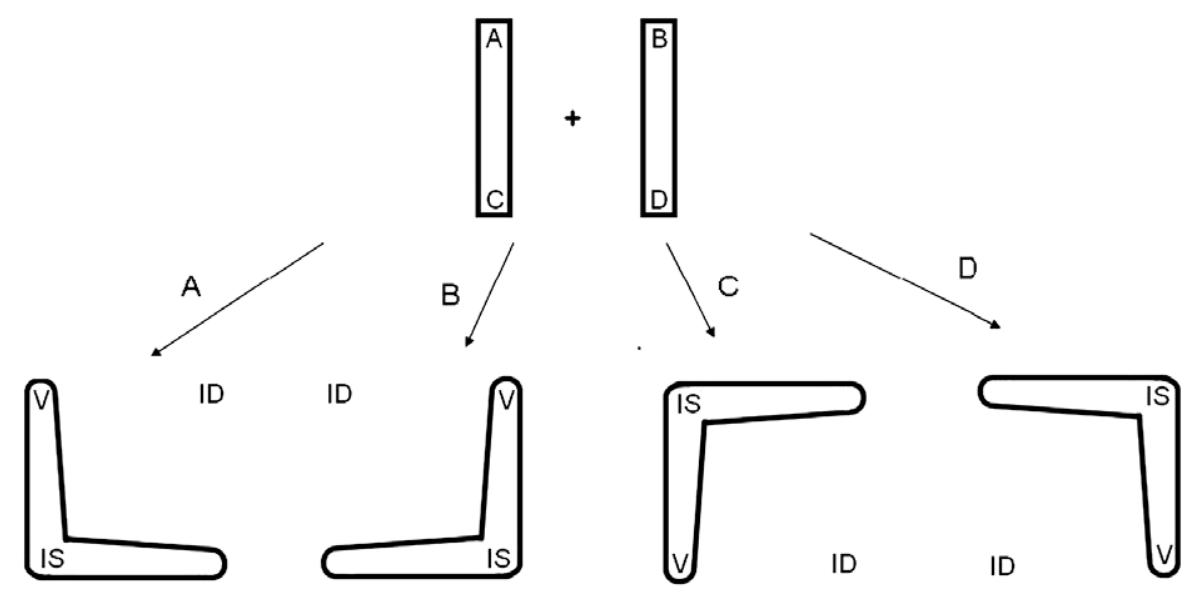

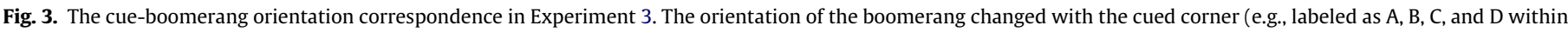
the rectangle). For example, when the cue appeared at A, the boomerang orientated as $L$. 
the rectangles, an object effect based on the boomerang orientation was always obtained, and was not affected by the cued rectangle. These results suggest that when the activated object (i.e., the cued rectangle) is disrupted, either by a sudden removal or replacement in a scene, attention does not seem to remain on it.

Lamy and Tsal (2000, Experiment 1) presented two similar objects (a rectangle and an hourglass) in different colors and then, before the target was shown, swapped the cued object and the uncued object to decouple the location and the colored shape of the two objects. After the switch, detection of the target at the previously cued object location (i.e., the invalid-same trials with respect to the cued object, with the location now occupied by the uncued object) was faster than at the previously uncued object location (which was now occupied by the cued object), implying that it was the grouped location belonging to the cued object (including cued and uncued locations of the previously attended object), but not the colored shape of the cued object, that was selected. Our finding shows that attention seems to select only the cued location, but not the uncued, supposedly invalid-same, location that was previously occupied by the cued object (i.e., there is no object effect of this sort in Experiments 1 and 2.) This seems contradictory to the findings of Lamy and Tsal.

One possible account which could reconcile Lamy and Tsal's (2000) (Experiment 1) result and ours is that when the cued object changes suddenly, the cued location remains operational (Brown \& Denney, 2007) and it may be used to connect with the new, uncued, object. That is, after the sudden swap between the cued object (e.g., a rectangle) and the uncued object (e.g., an hourglass) in Lamy and Tsal's study (2000), attention may quickly be reallocated to the uncued replacing object (the hourglass), with the help of the cued location. Faster responses when the target and the cue appeared at the same location than when at other locations (i.e., the location effect) in both their study and ours indicated this possibility, and the majority of their participants also saw the originally cued object being replaced with a different object. However, such a possibility could not be tested in Lamy and Tsal's study, because the objects in their study were too similar to determine whether attention remained at the location occupied previously by the cued object or was reallocated to the location occupied by the uncued new object. We could differentiate these two possibilities and showed that after a sudden removal or replacement of the object, attention was quickly reallocated either to the empty location after a removal, resulting in no object effect, or to the location occupied by the uncued replacing object, influencing the occurrence of the object effect. This seems to support the view that location is unique in selective attention (e.g., Kim \& Cave, 1995; Kramer, Weber, \& Watson, 1997; Lavie \& Tsal, 1994), and we further extended this idea by showing that it is unique in connecting space- and object-based attention. Also, the reduced location effect when the original objects (the rectangles) were replaced with a new object (a boomerang) in our Experiment 2 also indicates the interplay between space- and object-based attention, since the former is also affected by the latter.

In addition to attentional reallocation, other, not mutually exclusive, explanations could also account for the current results. It is possible that the feedback pathways from higher cortical levels guided attention less efficiently when the rectangles in the target frame were removed in Experiment 1. The appearance of the objects in the target frame could provide the visual system with the instantaneous and detailed properties of the external inputs (e.g., orientation, contour, color, position, and so on; see Cave \& Batty, 2006, for a review), transferred up to the higher cortical levels (Posner \& Raichle, 1994). The top-down processes would access those at lower levels (Ahissar \& Hochstein, 2004) in terms of current behavioral goal and past experience. The sudden onset of a target (a grey square) in the empty space may also immediately capture attention (e.g., Yantis, 1993). As a result, without the constraint of the objects (e.g., uniform connectedness and closure in our study), a sudden target onset in a blank display that captures attention to an empty space may have caused the absence of the object effect. Along the same line, the sudden onset of the boomerang in Experiments 2 and 3 may also capture attention involuntarily, indicating the operation of bottom-up constraints on the object effect. Consistent with the dynamic updating hypothesis, it is this new object that determines the object effect, rather than the originally cued object.

The second finding is that across-trial experience of seeing the boomerang plays an important role in the object effect we observed here. We showed that this experience of having seen the boomerang could influence the object effect of the rectangles in Experiments 2 and 3 . Note that we do not exclude the possibility that bottom-up constraints (e.g., the uniform connectedness and closure of the rectangles) may have played some role as well. It is the combination of the two which causes the net effect: the absence of the object effect rather than opposite object effect (i.e., faster for the invalid-different trials than for the invalid-same trials). The correspondence in cue and boomerang orientation may also contribute to the relatively smaller location effect when the boomerang appeared in Experiment 2 than in Experiment 3, and than when the unchanged rectangles displayed in Experiment 1 ( $p s<.001)$. In Experiment 2, the invalid same-object target location on the rectangle was always diagonal to the invalid same-object target location on the boomerang (see Fig. 2). When the rectangle was cued, attention may have been predisposed to shift to the invalid-different object target location on the uncued rectangle (i.e., the invalid same-object target location on the boomerang), due to the across-trial experience of seeing the boomerang. Therefore, when the boomerang suddenly replaced the rectangles, a target on the invalid same-object target location on the boomerang could be detected quickly, and this, in turn, produced a relatively smaller location effect since attention has, presumably, been shifted away from the cued location already. It is the interaction of the presence of a new object and the object's particular form that determines the location effect in Experiment 2.

The third finding is that the manipulated probability of an object's presence could affect the overall patterns of the target detection RT, but had no influence on the occurrence of the object effect. Target detection RT depended on both the probability of the object's presence ( $80 \%$ vs. $20 \%$ ) and what was shown in the target frame (sustained rectangles vs. disappearing ones in Experiment 1 , and rectangles vs. the boomerang in Experiments 2 and 3). Because it takes time to orient to the suddenly changed stimulus display (e.g., removal of rectangles in Experiment 1 and appearance of boomerang in Experiments 2 and 3), it follows that it thus takes longer to detect the target when the cued object was changed, and this advantage of the unchanged displays over the changed displays interacted with expectation (object probability): the RT difference between the changed and unchanged display was larger when the change was unexpected than when it was expected.

Why does the probability of object presence affect overall patterns of target detection RT, but not the occurrence of the object effect? One possible explanation is that attention is captured by a sudden change in the target frame (an offset of the rectangles in Experiment 1 or an onset of the boomerang in Experiments 2 and 3) (Gellatly \& Cole, 2000; Yantis \& Jonides, 1984), rendering the manipulation of the expectation of object presence ineffective. As a result, searching for a target in such a scene may be influenced by the instantaneous change of the scene. Future studies could use a gradual change, instead of a sudden one, to test this hypothesis. Note that attentional capture by sudden change does not necessarily indicate a purely bottom-up process; top-down knowledge may also play a role (Atchley et al., 2000; Folk et al., 1992). 
Studies of top-down effects on OBA have led to mixed results. Some studies (e.g., Chen, 1998; Li \& Logan, 2008; Watson \& Kramer, 1999) have reported a top-down effect on OBA, whereas others have shown a null effect (e.g., Pratt \& Sekuler, 2001, and the current study). Chen (1998) reported that top-down subjective organization could overcome bottom-up object features (e.g., in her case, color), and influence the appearance of the object effect. Li and Logan (2008) showed that objects defined by skilled Chinese readers' lexical knowledge could influence the deployment of attention toward these objects (i.e., Chinese words). Finally, Watson and Kramer (1999) suggested that task demands (Experiment 2 ) and expectancy (Experiment 4) could influence the object effect. Because the current study also manipulated expectancy, we further discuss difference between this study and ours.

Watson and Kramer (1999) (Experiment 4) presented participants with a pair of wrenches and asked them to identify two targets (an open wrench end and a bent wrench end) that were either on the same wrench or on two different wrenches. They also manipulated the magnitude of concave discontinuity (smooth or sharp concavity). Typically, the object effect was not obtained when the concavity was sharp, because in this case participants selected from the parsed representations; namely, they treated the end and the body of the wrench as two different parts. Conversely, the object effect was obtained when the concavity was smooth, because participants selected from the single uniform representation of the whole wrench. In addition to bottom-up factors (concave discontinuity), Watson and Kramer asked whether expectancies on the level of representation (parsed vs. single,) which can change attentional selection, could influence the object effect. Immediately before presenting the pair of wrenches to which participants were to respond (the 'probe,'), Watson and Kramer presented another pair of wrenches (the 'prime'), which could also have either a smooth or a sharp concavity. They found that the type of 'prime' (smooth or sharp concavity) could influence the object effect based on the 'probe' wrenches, indicating an expectancy effect. That is, the object effect were obtained when the prime wrenches contained a smooth concavity and the probe wrenches contained a sharp concavity.

Attentional selection on object representations is a complex function of interactions between top-down and bottom-up factors, since various top-down factors can interact with each other and with bottom-up factors. Furthermore, top-down factors can be manipulated in many ways. For example, expectation can be manipulated by changing object-presence probability, as in the current study, or by altering the sequence in which stimuli are presented as in Watson and Kramer. Our study differs from Watson and Kramer's in that their expectation has an effect, but not ours. Since there are many design differences between Watson and Kramer's and our studies, there are many possibilities that can account for this discrepancy. One possibility is that the sudden change in the target frame in our study (an offset of the rectangles in Experiment 1 or an onset of the boomerang in Experiments 2 and 3) may capture attention, rendering the manipulation of the expectation of an object's presence ineffective. In Watson and Kramer's study, the probe wrenches did not undergo sudden change.

Another difference is the paradigms used. Watson and Kramer (1999) adopted the divided-attention paradigm, whereas we used the cuing paradigm. The divided-attention paradigm was first introduced by Duncan (1984), who asked participants to report two features that belonged either to the same object or to two different objects. Typically, the proportion of correct responses is greater when the two features are in the same object rather than in two different objects. OBA has been demonstrated many times in the divided-attention paradigm for different object types (e.g., Kramer et al., 1997; Lavie \& Driver, 1996), for occluded objects (e.g., Behrmann, Zemel, \& Mozer, 1998), for parts of objects (e.g.,
Barenholtz \& Feldman, 2003), and for texton-based segregation (Ben-Shahar, Scholl, \& Zucker, 2007). However, since there is no cue in the divided-attention paradigm, it does not help us in examining the cued object hypothesis. Although studies using the divided-attention paradigm indicate that cueing is not necessary for the object effect, the divided-attention and cuing paradigms may have different levels of sensitivity in measuring OBA (Marino \& Scholl, 2005) and the specific processes involved, despite the fact that both paradigms provide converging evidence that, in general, attention could be allocated to objects in a way very similar to spatial attention.

In the current study, when the rectangles persisted throughout a trial, the object effect was about $10 \mathrm{~ms}$ to $13 \mathrm{~ms}$. The rectanglebased object effect was computed by comparing invalid same-object target RTs and invalid-different object target RTs (i.e., objectto-object). However, when the boomerang replaced the rectangles in the target frame, the object effect based on this boomerang was about $30 \mathrm{~ms}$ to $50 \mathrm{~ms}$. The boomerang-based object effects were computed by comparing invalid same-object RTs and invalid outof-object target RTs (i.e., object-to-space). These different sizes of the object effect may give us some insights on the underlying mechanisms of the object effect. There are at least two primary mechanisms: processing advantage within the cued object (attentional spreading, e.g., Abrams \& Law, 2000, and scanning prioritization, e.g., Shomstein and Yantis, 2004) and attentional disengagement from the cued object (e.g., Brown and Denney, 2007). The different sizes of the object effect based on either the rectangles or the boomerang could be explained by both accounts.

Processing advantage within the cued object indicates that the object effect is due to either attentional enhancement or scanning priority within the entire cued object. According to this account, when the rectangles persisted throughout the trial, attention could enhance the representational quality of the cued rectangle (attentional spreading) or scan it with highest priority (scanning prioritization). This would result in the difference in RT between the invalid within-object and invalid between-object target conditions. Similarly, when the boomerang replaced the rectangles in the target frame, attention could enhance the representational quality of the boomerang or scan it with highest priority, causing a difference in RT between the invalid within-object and invalid object-tospace target conditions. Because the boomerang is larger than one rectangle, attention may spend more time spreading or scanning the boomerang. Consequently, the RT difference when the boomerang interrupted the rectangles should be larger than that when the rectangles persisted. On the other hand, attentional disengagement from the cued object indicates that the object effect may be due to a difficulty in disengaging from the cued object. It is possible that attention somehow is more difficult to disengage from the boomerang, thus causing a larger object effect.

Figure-ground segregation could also affect the object effect. The rectangles and boomerang could be seen as figures, which could be processed in higher priority as compared to the ground. Seen from this perspective, the target in Experiments 2 and 3 would have appeared on a figure in the invalid-same object condition but on the ground in the invalid-different object condition when the boomerang appeared, while the target in Experiment 1 appeared in the figures when the rectangles persisted; this could explain the larger object effect found in Experiment 1 than in Experiments 2 and 3. Moreover, when the rectangles (two figures) persisted throughout the trial, attention may have enhanced the uncued figure to some extent, or attention could have quickly shifted to search for the uncued figure. However, when the boomerang (one figure) appeared, attention may not have enhanced the spatial area out of the boomerang (ground) or attention may not have quickly shifted to search for the location belonging to the background. More studies could be conducted to investi- 
gate how the figure-ground segregation influences the object effect.

In conclusion, the results of the three sets of experiments in this study showed that bottom-up instantaneous object inputs and topdown constraints such as experience of previewing another object combine to affect the object effect. When an attended object disappears suddenly or when a new object appears abruptly in the target frame, it is the updated object that determines attentional selection, while having seen another object across trials also affects how attention operates on the cued object.

\section{Acknowledgments}

This research was supported by grants from the National Science Council in Taiwan (NSC94-2811-H-002-002, NSC95-2413-H002-020, and NSC96-2752-H-002-008-PAE). We thank Zhe Chen, Morris Goldsmith, and one anonymous reviewer for their helpful comments, and Yi-Jia Su for her assistance in conducting part of the experiments.

\section{References}

Abrams, R. A., \& Law, M. B. (2000). Object-based visual attention with endogenous orienting. Perception \& Psychophysics, 62, 818-833.

Ahissar, M., \& Hochstein, S. (2004). The reverse hierarchy theory of visual perceptual learning. Trends in Cognitive Sciences, 8, 457-464.

Atchley, P., Kramer, A. F., \& Hillstrom, A. P. (2000). Contingent capture for onsets an offsets: Attentional set for perceptual transients. Journal of Experimental Psychology: Human Perception and Performance, 26, 594-606.

Bacon, W. F., \& Egeth, H. E. (1994). Overriding stimulus-driven attentional capture Perception \& Psychophysics, 55, 485-496.

Barenholtz, E., \& Feldman, J. (2003). Perceptual comparisons within and between object parts: Evidence for a single-object superiority effect. Vision Research, 43 1655-1666.

Behrmann, M., Zemel, R., \& Mozer, M. (1998). Object-based attention and occlusion: Evidence from normal participants and a computational model. Journal of Experimental Psychology: Human Perception and Performance, 24, 1011-1036.

Ben-Shahar, O., Scholl, B. J., \& Zucker, S. W. (2007). Attention, segregation, and textons: Bridging the gap between object-based attention and texton-based segregation. Vision Research, 47, 845-860.

Brown, J. M., \& Denney, H. I. (2007). Shifting attention into and out of objects: Evaluating the processes underlying the object advantage. Perception $\mathcal{E}$ Psychophysics, 69, 606-618.

Cave, K. R., \& Batty, M. J. (2006). From searching for features to searching for threat: Drawing the boundary between preattentive and attentive vision. Visual Cognition, 14, 629-646.

Chen, Z. (1998). Switching attention within and between objects: The role of subjective organization. Canadian Journal of Experimental Psychology, 52, 7-16.

Downing, P. E. (2000). Interactions between visual working memory and selective attention. Psychological Science, 11, 467-473.

Duncan, J. (1984). Selective attention and the organization of visual information. Journal of Experimental Psychology: General, 113, 501-517.

Egly, R., Driver, J., \& Rafal, R. D. (1994). Shifting visual attention between objects and locations: Evidence from normal and parietal lesion subjects. Journal of Experimental Psychology: General, 123, 161-177.

Eriksen, C. W., \& Yeh, Y. Y. (1985). Allocation of attention in the visual field. Journa of Experimental Psychology: Human Perception and Performance, 5, 583-597.

Farah, M. J. (1985). Psychophysical evidence for a shared representational medium for mental images and percepts. Journal of Experimental Psychology: General, 114 93-103.
Farah, M. J. (1989). Mechanisms of imagery-perception interaction. Journal of Experimental Psychology: Human Perception and Performance, 15, 203-211.

Folk, C. L., \& Remington, R. W. (1999). Can new objects override attentional control settings? Perception \& Psychophysics, 61, 727-739.

Folk, C. L., Remington, R. W., \& Johnston, J. C. (1992). Involuntary covert orienting is contingent on attentional control settings. Journal of Experimental Psychology: Human Perception and Performance, 18, 1030-1044.

Gellatly, A., \& Cole, G. (2000). Accuracy of target detection in new-object and oldobject displays. Journal of Experimental Psychology: Human Perception and Performance, 26, 889-899.

Goldsmith, M., \& Yeari, M. (2003). Modulation of object-based attention by spatial focus under endogenous and exogenous orienting. Journal of Experimental Psychology: Human Perception and Performance, 29, 897-918.

Jefferies, L. N., Wright, R. D., \& Di Lollo, V. (2005). Inhibition of return to an occluded object depends on expectation. Journal of Experimental Psychology: Human Perception and Performance, 31, 1224-1233.

Kim, M. S., \& Cave, K. R. (1995). Spatial attention in visual search for features and feature conjunctions. Psychological Science, 6, 376-380.

Kramer, A. F., Weber, T. A., \& Watson, S. E. (1997). Object-based attentional selection-grouped arrays or spatially invariant representations? Comment on Vecera and Farah (1994). Journal of Experimental Psychology: General, 126, 3-13.

Lamy, D., \& Tsal, Y. (1999). A salient distractor does not disrupt conjunction search. Psychonomic Bulletin \& Review, 6, 93-98.

Lamy, D., \& Tsal, Y. (2000). Object features, object locations, and object files: Which does selective attention activate and when? Journal of Experimental Psychology: Human Perception and Performance, 26, 1387-1400.

Lavie, N., \& Driver, J. (1996). On the spatial extent of attention in object-based selection. Perception \& Psychophysics, 58, 1238-1251.

Lavie, N., \& Tsal, Y. (1994). Perceptual load as a major determinant of the locus of selection in visual attention. Perception $\mathcal{E}$ Psychophysics, 56, 183197

Li, X., \& Logan, G. D. (2008). Object-based attention in Chinese readers of Chinese words: Beyond Gestalt principles. Psychonomic Bulletin \& Review, 15, 945-949.

Marino, A. C., \& Scholl, B. J. (2005). The role of closure in defining the 'objects' of object-based attention. Perception \& Psychophysics, 67, 1140-1149.

Miller, J. (1989). The control of attention by abrupt visual onsets and offsets. Perception \& Psychophysics, 45, 567-571.

Posner, M. I., \& Raichle, M. E. (1994). Images of mind. New York: W.H. Freeman and Company.

Posner, M. I., Snyder, C. R. R. \& Davidson, B. J. (1980). Attention and the detection of signals. Journal of Experimental Psychology: General, 109, 160-174.

Pratt, J., \& Sekuler, A. B. (2001). The effects of occlusion and past experience on the allocation of object-based attention. Psychonomic Bulletin E Review, 8, 721-727.

Schneider, W., Eschman, A., \& Zuccolotto, A. (2002). E-prime user's guide. Pittsburgh, PA: Psychology Software Tools

Scholl, B. J. (2001). Objects and attention: The state of the art. Cognition, 80, 1-46.

Shomstein, S., \& Yantis, S. (2002). Object-based attention: Sensory modulation or priority setting? Perception \& Psychophysics, 64, 41-51.

Shomstein, S., \& Yantis, S. (2004). Configural and contextual prioritization in objectbased attention. Psychonomic Bulletin \& Review, 11, 247-253.

Vecera, S. P. (1994). Grouped locations and object-based attention: Comment on Egly, Driver, and Rafal (1994). Journal of Experimental Psychology: General, 123, 316-320.

Vecera, S. P., \& Farah, M. J. (1994). Does visual attention select objects or locations? Journal of Experimental Psychology: General, 123, 146-160.

Watson, S. E., \& Kramer, A. F. (1999). Object-based visual selective attention and perceptual organization. Perception E Psychophysics, 61, 31-49.

Yantis, S. (1993). Stimulus-driven attentional capture and attentional control settings. Journal of Experimental Psychology: Human Perception and Performance, 19, 676-681.

Yantis, S., \& Jonides, J. (1984). Abrupt visual onsets and selective attention: Evidence from visual search. Journal of Experimental Psychology: Human Perception and Performance, 10, 601-621.

Yeh, S. L., \& Liao, H. I. (2008). On the generality of the contingent orienting hypothesis. Acta Psychologica, 129, 157-165. 